\title{
Diaphragmatic Hernia with Gastric Volvulus and Complete Gastric Outlet Obstruction
}

\author{
Hayder Al-Masari, M.D., Heba Nofal, M.D., Rawan Majdalawi, M.D., Reham Ainawi, M.D., Abdulwahid Alwahedi, M.D., \\ Tarek Mahdi, M.D., Ph.D. \\ Bariatric Unit, General Surgery Department, Al-Qassimi Hospital, Sharjah, United Arab Emirates
}

A combination of Cytoreductive surgery (CRS) along with hyperthermic intraperitoneal chemotherapy (HIPEC) considers a crucial approach in treating designated patients with alimentary and gynecological malignancies with the involvement of the peritoneal cavity. The foremost frequent surgical complications are leakage, digestive perforations, fistulas, intestinal obstruction, abscess, and peripancreatitis. This report presents a case of a patient with a late acquired herniation of guts through the diaphragm after CRS and HIPEC that were already done 6 months back. A 26 -year- old male previously treated with CRS and HIPEC for testicular mesothelioma with peritoneal involvement, was admitted to our unit with the diagnosis of gastric outlet obstruction. His CT scan illustrated a left diaphragmatic hernia involving the stomach and splenocolic flexure. Each denudation of the diaphragmatic serosa throughout CRS which typically occurs during the surgical operation in a combination of the HIPEC heat can explain such complication. The herniation is extremely uncommonly diagnosed after CRS and HIPEC. Surgical techniques for hernia repair can be done by direct suturing of the defect or closure with artificial or biological tissue, each technique is a potential surgical technique for repair with reliable long-run results.

Keywords: Diaphragmatic hernia, HIPEC, Cytoreductive surgery

This is an Open Access article distributed under the terms of the Creative Commons Attribution Non-Commercial License (http:// creativecommons.org/licenses/by-nc/4.0/) which permits unrestricted non-commercial use, distribution, and reproduction in any medium, provided the original work is properly cited.

\author{
Received April 24, 2020 \\ Revised September 19, 2020 \\ Accepted October 14, 2020 \\ Corresponding author \\ Hayder Al-Masari \\ Bariatric Unit, General Surgery \\ Department, Al-Qassimi Hospital, \\ Tawuun Street, Al-Sham Tower, 901, \\ Sharjah 61111, United Arab Emirates \\ Tel: $+971-566298515$ \\ Fax: +971-65387200 \\ E-mail:Hm.hamadi@mohap.gov.ae \\ ORCID: \\ https://orcid.org/0000-0002-9317-1006
}

\section{INTRODUCTION}

Diaphragmatic hernia post-CRS and HIPEC surgeries for some gastrointestinal and gynecological tumors are rare but it could be a life-threatening complication that needs efficient and prompt intervention and management.

Diaphragmatic hernia (DH) is a defect within the diaphragm that leads to protrusion of abdominal contents and organs into the thoracic cavity. It can be a congenital hernia, which is the most common type, however, it can be acquired. ${ }^{1}$ There are various causes for acquired diaphragmatic hernia but mostly it occurs following blunt or penetrating trauma which leads to diaphragmatic rupture and as a sequelae herniation of the abdominal content may follow. Moreover, there are cases reported that acquired $\mathrm{DH}$ can also occur by iatrogenic causes or spontaneously. $^{2}$

Complications of diaphragmatic hernia include acute obstructive symptoms, diaphragmatic rupture, cardiac tamponade, respiratory failure, strangulation, and incarceration. ${ }^{1,3}$ Even though, acquired DH is a rare situation but it can be considered a life-threatening situation when incarceration and strangulation occurred with nearly an overall mortality reach up to $31 \%$.

In acute settings, the treatment of acquired diaphragmatic hernia needs efficient resuscitation to the patient. ${ }^{2}$ The next step in the management is surgical which is typically done by primary closure, ${ }^{5}$ yet, in cases where primary closure with non-absorbable sutures is not possible an alternative with mesh repair can be used due to the size of the defect. ${ }^{5}$ 
Peritoneal carcinomatosis that could be a result of gynecological or colorectal malignancies has been considered in the past as a lethal clinical situation, also had been regarded as an exclusion criterion for performing resective surgery. ${ }^{6}$ Parietal and visceral peritonectomy as a cytoreductive approach along with hyperthermic intraperitoneal chemotherapy (HIPEC) is an efficient treating approach for selected cases with gastrointestinal and gynecological malignancies having peritoneal spread.

The main purpose of cytoreductive surgery (CRS) is to achieve complete macroscopic cytoreduction (CC-0/1) as a precondition to be able to apply HIPEC. The classification of residual disease is mainly obtained intraoperatively by using the completeness of cytoreduction (CCR) score. When mentioning CCR-0 this will indicate no visible residual tumor, moreover; CCR-1 residual tumor nodules $2.5 \mathrm{~mm}$, CCR-2, and CCR-3 indicate residual tumor nodules between $2.5 \mathrm{~mm}$ and $2.5 \mathrm{~cm}$ and equal or more than $2.5 \mathrm{~cm}$, respectively. If we have a score of CCR 0 or 1 , the performance of HIPEC can through an open or closed abdomen approach. ${ }^{7}$ To circulate the chemotherapy a special pump is needed via several catheters applied into the peritoneal cavity. It's important to keep the intraperitoneal temperature in between $41^{\circ} \mathrm{C}$ to $43^{\circ} \mathrm{C}$. We can use several drugs for HIPEC for instance: (Cisplatinum, mitomycin C, oxaliplatin, irinotecan, etc.). ${ }^{8}$ After the operation, the most frequent complications that can happen are mainly abscesses, anastomotic leakage, fistulas, and digestive perforations. A review of the literature showed that only three patients had diaphragmatic hernia secondary to CRS. ${ }^{9}$

\section{CASE REPORT}

A 27-year-Emirates, college graduate man, no previous medical history, was previously diagnosed with left testicular mesothelioma in October 2018, who underwent cytoreductive surgery with hyperthermic intraperitoneal chemotherapy (CRS-HIPEC) in April 2019 with 4 cycles of chemotherapy (Cisplatin, Pemetrexed, and Bevacizumab), referred to the Department of General Surgery in Al Qassimi Hospital in September 2019, due to repeated vomiting with sudden severe abdominal distension and absolute constipation and failure to thrive. On Physical examination, the patient was afebrile, Pulse rate 112, maintaining normal blood pressure 126/78, and he was dehydrated. The abdomen was distended with a positive bowel sound, on chest auscultation, there was diminished air entry on the left chest wall. Furthermore, investigations showed, WBC count was 16.5, CRP 215, and Hg level was 15 . Resuscitation is done with 2 wide bore iv cannulas and NG tube insertion. CXR was done and it showed gastric shadow with air-fluid level herniated through the left hemidiaphragm, for further investigations. Followed by a gastrografin swallow study through the NG tube, it showed distortion of the gastric anatomy with organoaxial volvulus and complete gastric outlet obstruction (Fig. 1).

After resuscitation, the patient as taken for emergent surgery which was started by laparoscopic exploration where the stomach was found upside down in the thorax, with part of small bowel and colon, and evidence of partial ischemia of the stomach wall. Later on, the surgery was converted to open laparotomy due to the incarceration of the stomach in the thorax, as it was not possible to be pulled down in the abdominal cavity due to adhesion to the left lung tissue and despite extending the hernia wall in the diaphragm (Figs. 2 5).

A midline laparotomy was done, the abdominal cavity was opened, then reduction of the herniated organs from the chest cavity was done, later the diaphragmatic defect was closed in two-layer with non-absorbable sutures and a protective gastrostomy tube was placed and fixed to the abdominal wall. Then irrigation was done with Dermacyn and a left-sided chest tube was inserted under vision.

Finally, closure of the abdominal wall was done in layers and then the skin was closed by with skin clips.

\section{DISCUSSION}

Combination therapy of CRS and HIPEC in selective patients with peritoneal spread with gastrointestinal and gynecological malignancies became a new feasible therapy that achieves promising results in terms of prognosis, with an acceptable morbidity rate, compared to palliative systemic chemotherapy in the past in patients with regional intraperitoneal cancer spread. ${ }^{6}$ The primary goal of this therapy is to resect as much as possible of the

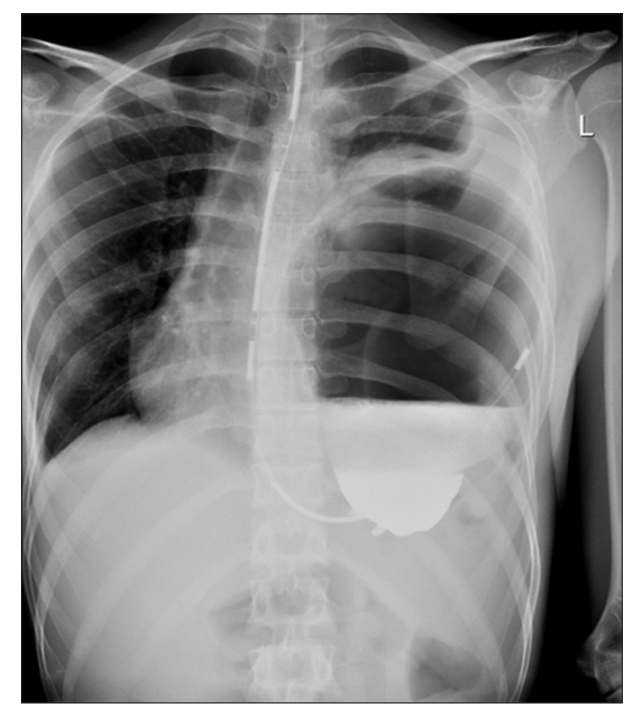

Fig. 1. A large cystic structure is seen with air-fluid level, with NG tube seen inside suggesting distended stomach suspected pushing the diaphragm upward. 


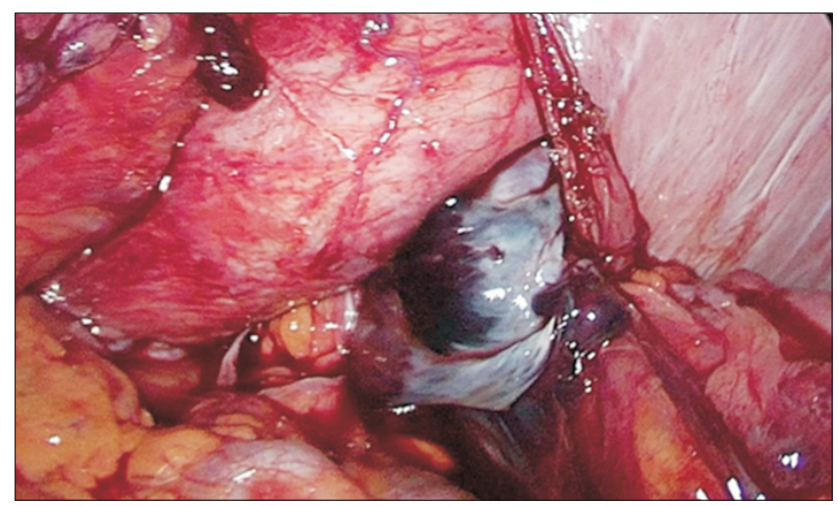

Fig. 2. Tight diaphragmatic defect with evidence of partial ischemia of the stomach wall and part of small bowel and colon herniating through the defect.

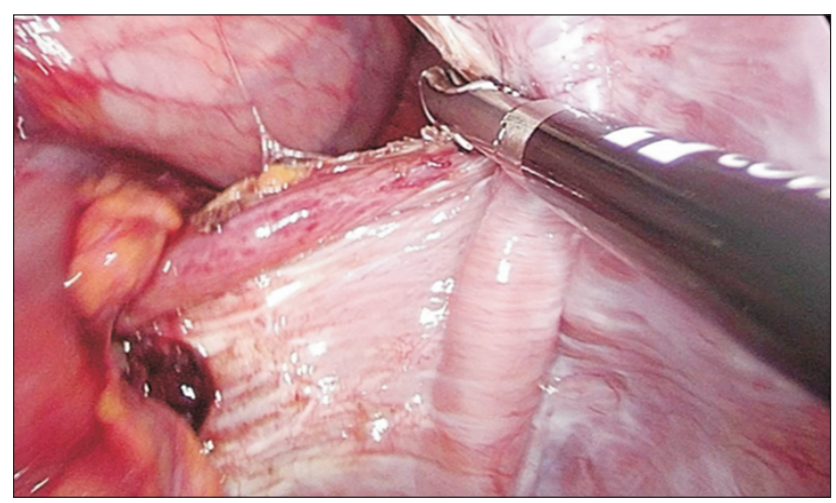

Fig. 3. Extension of diaphragmatic defect to allow content reduction.

macroscopic tumor and to destruct the remaining microscopic tumor by using cytotoxic chemotherapeutic agents and hyperthermia. ${ }^{6}$

However, the most frequent postoperative complications associated with this combination therapy are anastomotic leakage, digestive perforations, fistulas, intestinal obstruction, abscess, and peripancreatitis. Yet, diaphragmatic hernia post-CRS and HIPEC therapy is rarely diagnosed. In the review of the literature, only three cases of diaphragmatic hernia post-CRS and HIPEC therapy are reported. ${ }^{9}$ The first post-surgical complication described in the literature was reported by Laterza et al. in 2009 , in a 70-year-old patient diagnosed with malignant mesothelioma of the peritoneum. The patient developed a colobronchial fistula which besides was aided by a diaphragmatic defect after CRS and HIPEC therapy.

In the literature review reported by Youssef $\mathrm{H}$ et al. in 2005, where the Kaplan-Meier statistical method showed that 5- and 10 -years overall survival predicted was $69 \%$ and $57 \%$, respectively. Furthermore, in a comparison between 289 patients who underwent complete cytoreduction where the 5 and 10 -year pre-

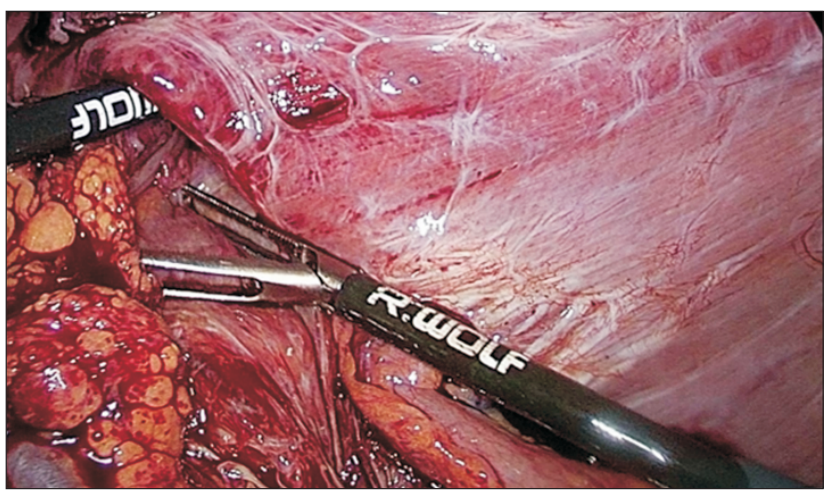

Fig. 4. Post-trial reduction of the defect during laparoscopic surgery.

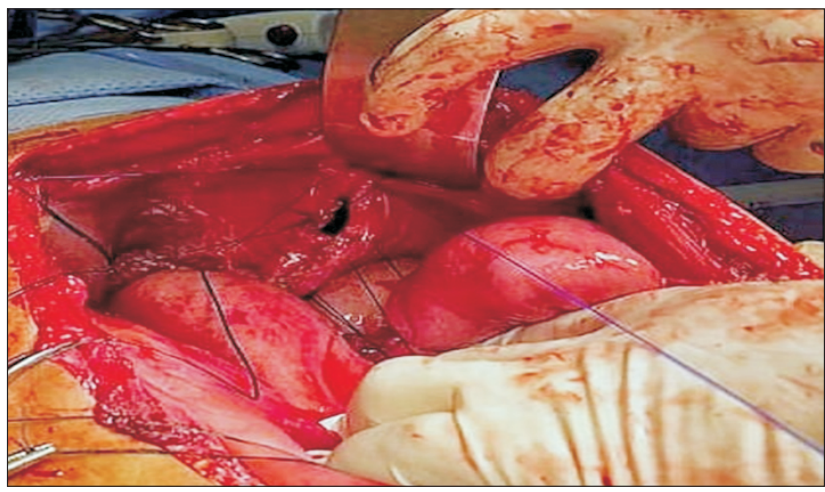

Fig. 5. Content reduction and diaphragmatic repair after open laparotomy.

dicted survival was $87 \%$ and $74 \%$ and 152 patients who had major tumor debulking, predicted survival was respectively $34 \%$ and $23 \% .^{10}$

Parietal peritonectomy surgery in the area of the diaphragm by using the cautery can partially injure the muscular tissue, resulting in possible adjacent lesions with a thin muscular layer that can lead to subsequent defect formation due to electrocoagulation and hyperthermia effects as it considered a primary responsible cause. Furthermore, prolongation of the healing of the defect by the cytotoxic effect of the HIPEC and elevated abdominal pressure during HIPEC which will further stretch the diaphragm can further contribute in hernia development.

The definitive treatment for a diaphragmatic hernia is surgery, where urgent surgery is only for symptomatic patients compared with asymptomatic patients where surgery can be delayed days or years later according to the patient's status. Primary closure with non-absorbable sutures is the main principle in repairing the small diaphragmatic defects with interrupted stitches is the best way to close the small defect. Taking into consideration tension-free closure with maintaining good blood supply to the margins. While in a case with large diaphragmatic defect or muscle weakness, the synthetic or biological xenogeneic materi- 
als is the best way for repairing as the primary repair can lead to excessive tension. Also, mesh placement between the layers of the peritoneum should be considered because adhesions between the synthetic graft and organs can result after an operation, thereby in the patient that underwent CRS with total diaphragmatic peritonectomy mesh should not be used. ${ }^{6}$

\section{CONCLUSION}

Even though diaphragmatic hernia complication is rarely diagnosed with post-CRS and HIPEC therapy compared with other postoperative complications, it should be considered when preforming peritonectomy, especially in the upper quadrant in association with HIPEC. Whenever this complication is encountered surgery is the only best choice for symptoms resolution and for a better quality of life.

\section{ETHICAL STATEMENT}

This study was exempted from review by the Dubai Research Ethics Committee of the Ministry of Health and Prevention.

\section{ORCID}

Hayder Al-Masari, https://orcid.org/0000-0002-9317-1006

Heba Nofal, https://orcid.org/0000-0002-3576-4129

Rawan Majdalawi, https://orcid.org/0000-0001-9585-9683

Reham Ainawi, https://orcid.org/0000-0003-3728-5057

Abdulwahid Alwahedi, https://orcid.org/0000-0002-0153-3525

Tarek Mahdi, https://orcid.org/0000-0003-1302-9024

\section{AUTHORS' CONTRIBUTIONS}

Conceptualization: Hayder Al-Masari, Heba Nofal, and Abdulwahid Alwahedi. Formal analysis: Hayder Al-Masari, Heba Nofal, and Tarek Mahdi. Methodology: Hayder Al-Masari, Tarek Mahdi, and Abdulwahid Alwahedi. Writing-original draft: Heba Nofal, Rawan Majdalawi, and Reham Ainawi. Writing-review and editing: Hayder Al-Masari and Heba Nofal.

\section{CONFLICT OF INTEREST}

None.

\section{FUNDING}

None.

\section{ACKNOWLEDGMENTS}

The authors would like to thank the patient for his participation and his consent to the publication of the case details and associated images.

\section{REFERENCES}

1) Chandrasekharan PK, Rawat M, Madappa R, Rothstein DH, Lakshminrusimha S. Congenital Diaphragmatic hernia - a review. Matern Health Neonatol Perinatol 2017;3:6.

2) Katukuri GR, Madireddi J, Agarwal S, Kareem H, Devasia T. Delayed Diagnosis of Left-Sided Diaphragmatic Hernia in an Elderly Adult with no History of Trauma. J Clin Diagn Res 2016;10:Pd04-05.

3) D’Orio V, Demondion P, Lebreton G, Coutance G, Varnous S, Leprince $\mathrm{P}$. Acquired transdiaphragmatic hernia: an unusual cause of cardiac tamponade. Asian Cardiovasc Thorac Ann 2017;25:233-236.

4) Lu J, Wang B, Che X, et al. Delayed traumatic diaphragmatic hernia: A case-series report and literature review. Medicine (Baltimore) 2016;95:e4362

5) Eren S, Ciriş F. Diaphragmatic hernia: diagnostic approaches with review of the literature. Eur J Radiol 2005;54:448-459.

6) Sorrentino L, Cabry F, Serra F, Gelmini R. A rare case of diaphragmatic hernia after cytoreductive surgery and hypertermic intraperitoneal chemotherapy. J Peritoneum (and other serosal surfaces) 2017;2:95-98.

7) Jacquet $P$, Sugarbaker PH. Clinical research methodologies in diagnosis and staging of patients with peritoneal carcinomatosis. Cancer Treat Res 1996;82:359-374.

8) Glehen O, Cotte E, Kusamura S, et al. Hyperthermic intraperitoneal chemotherapy: nomenclature and modalities of perfusion. J Surg Oncol 2008;98:242-246.

9) Lampl B, Leebmann H, Mayr M, Piso P. Rare diaphragmatic complications following cytoreductive surgery and HIPEC: report of two cases. Surg Today 2014;44:383-386.

10) Youssef H, Newman C, Chandrakumaran K, Mohamed F, Cecil TD, Moran BJ. Operative findings, early complications, and long-term survival in 456 patients with pseudomyxoma peritonei syndrome of appendiceal origin. Dis Colon Rectum 2011;54:293-299. 